\title{
Applying different mathematical variability methods to identify older fallers and non-fallers using gait variability data
}

\author{
Nise Ribeiro Marques ${ }^{1,5}$ - Camilla Zamfolini Hallal ${ }^{2} \cdot$ Deborah Hebling Spinoso $^{1}$. \\ Mary Hellen Morcelli ${ }^{1} \cdot$ Luciano Fernandes Crozara $^{3} \cdot$ Mauro Gonçalves $^{4}$
}

Received: 21 February 2016/Accepted: 18 May 2016/Published online: 2 June 2016

(C) Springer International Publishing Switzerland 2016

\begin{abstract}
Background The clinical assessment of gait variability may be a particularly powerful tool in the screening of older adults at risk of falling. Measurement of gait variability is important in the assessment of fall risk, but the variability metrics used to evaluate gait timing have not yet been adequately studied.

Objectives The aims of this study were (1) to identify the best mathematical method of gait variability analysis to discriminate older fallers and non-fallers and (2) to identify the best temporal, kinematic parameter of gait to discriminate between older fallers and non-fallers.

Methods Thirty-five physically active volunteers participated in this study including 16 older women fallers $(69.6 \pm 8.1$ years) and 19 older women non-fallers $(66.1 \pm 6.2$ years). Volunteers were instructed to walk for $3 \mathrm{~min}$ on the treadmill to record the temporal kinematic gait parameters including stance time, swing time and stride time by four footswitches sensors placed under the volunteers' feet. Data analysis used 40 consecutive gait cycles. Six statistical methods were used to
\end{abstract}

Nise Ribeiro Marques

nisermarques@yahoo.com.br

1 Department of Physical Therapy and Occupational Therapy, São Paulo State University, UNESP, Marília, Brazil

2 Department of Physical Education and Physical Therapy, Uberlândia Federal University, UFU, Uberlândia, Brazil

3 Faculty of Medicine of Marilia, FAMEMA, Marília, Brazil

4 Department of Physical Education, São Paulo State University, UNESP, Rio Claro, Brazil

5 Departamento de Fisioterapia e Terapia Ocupacional, UNESP, Avenida Hygino Muzzi Filho, 737, Marília, SP 14325-000, Brazil determine the variability of the stance time, swing time and stride time. These included: (1) standard deviation of all the time intervals; (2) standard deviation of the means of these intervals taken every five strides; (3) mean of the standard deviations of the intervals determined every five strides; (4) root-mean-square of the differences between intervals; (5) coefficient of variation calculated as the standard deviation of the intervals divided by the mean of the intervals; and (6) a geometric method calculated based on the construction of a histogram of the intervals.

Results The standard deviation of 40 consecutive gait cycles was the most sensitive $(100 \%)$ and specificity $(100 \%)$ parameter to discriminate older fallers and nonfallers.

Conclusion The standard deviation of stance time is the kinematic gait variability parameter that demonstrated the best ability to discriminate older fallers from non-fallers. Protocol number of Brazilian Registry of Clinical Trials: RBR-6rytw2.

Keywords Aging $\cdot$ Falls risk $\cdot$ Kinematics

\section{Introduction}

Falls are the main cause of injury and injury-related death among older adults (65 years and older) accounting for $45 \%$ of all deaths in this population [1]. Falls contribute to decreased mobility, independence and quality of life. This can have high medical, personal and social costs $[2,3]$. There is an age-related progressive decline in sensory and motor function resulting in the impaired performance of complex motor tasks and an associated high rate of falls among older adults $[4,5]$. 
Approximately one-third of all older adults fall every year; $14 \%$ of older adults are recurrent fallers [6]. Thus, the ability to walk efficiently and safely is important for the maintenance of independence and health by reducing the risk of falls and fall-related injuries [7]. Reducing falls in older adults can be achieved by screening and early detection of the biomechanical changes in gait $[8,9]$. Researchers and clinicians often use kinematic gait analysis to identify changes in the motor pattern during walking [6]. Reduced gait speed and step length, increased double support phase, as well as reduced stride and step length are indicative of decreased mobility and increased risk of falls in older adults [10]. However, the measures of stride-to-stride variability in gait may be the best indicator of dynamic balance control and prospective risk of falling $[11,12]$.

The most common clinical parameters used to screen risk of falls are: functional scales (i.e., BERG Balance Scale), gait speed, average step length and step time, and gait variability [13]. Of these parameters, previous studies have shown that the average speed and step length and step time may be more related to fear of falling but not closely related to a risk of falls [13]. Thus, gait variability is more sensitive than other measures of gait analysis to provide a clinical assessment of gait instability and risk of falls in older adults [13].

Increased gait variability is a consequence of unstable and uneven gait, which make older adults more susceptible to falling [7, 14]. The variability in rhythmic physiological processes has been extensively studied in cardiology by analysis of the beat-to-beat time intervals of the heart. The heart rate and temporal-spatial gait parameters exhibit similar properties during their repetitive cycles, and it is possible to apply mathematical models developed in heart rate variability to gait variability [11]. Both heart rate and gait timing have regular and periodic behaviors over time like a metronome. However, there are several important differences in their interpretation because increased stride time variability is a sign of pathology, whereas increased heart rate variability is a sign of a healthy heart [11].

Despite the differences in heart rate variability and gait variability, the dynamic properties of both signals including the complex fluctuations reminiscent of fractals-this can be altered by aging [13]. Thus, the analysis and interpretation of gait variability on clinical practice may be enhanced by advancing methods and interpretation of these data such as applying methods of heart rate variability to analyze gait variability [13]. Therefore, there were two aims of this study: (1) identify the best mathematical method of gait variability analysis to discriminate older fallers and non-fallers and (2) identify the best temporal, kinematic parameter of gait to discriminate between older fallers and non-fallers.

\section{Methods}

\section{Participants}

We enrolled 35 physically active older women including 16 (69.6 \pm 8.1 years) with a history of falls and 19 $(66.1 \pm 6.2$ years $)$ without a history of falls. The participants were recruited from a community exercise group for older adults. Only women were included to eliminate sex as a confounder because women have a higher prevalence of falls than men [15].

The volunteers were classified as fallers based on a selfreport of falls in the previous 12 months [16]. A fall was defined as any balance perturbation, which caused the person's body to have significant contact with the floor [16]. If the participant had one or more fall episodes, then she was defined as a faller. Those who reported no falls were classified as non-fallers. Table 1 shows the characteristics of the study participants.

Subjects were excluded from the study for having musculoskeletal pain, fractures or severe soft tissue injury during the previous 6 months, or neurological, cardiovascular or respiratory diseases. The local ethics committee approved this study, and all participants signed an informed consent form.

\section{Instruments}

Kinematic data were collected using a telemetry data acquisition system and software for biological signals (Noraxon ${ }^{\circledR}$, Arizona, USA). The gait phases were determined using pressure sensors $\left(\right.$ Noraxon ${ }^{\circledR}$, Arizona, USA), which were sampled at $2000 \mathrm{~Hz}$. The footswitches were placed on the hallux, first metatarsal, fifth metatarsal and heel of the dominant leg. Gait analysis was performed while walking on a treadmill at the preferred gait speed (Inbramed ${ }^{\circledR}$, Porto Alegre, BRA). Overground preferred gait speed was determined using a photoelectric timing system (Speed Test 6.0, Campinas, BRA), and the subjects were instructed to walk at their self-selected pace.

\section{Procedures}

Initially, the preferred treadmill gait speed was determined based on the mean of the preferred 10-m overground walking speed over three consecutive trials [17]. After determining the preferred overground walking speed, the subjects walked on the treadmill starting at a speed of $50 \%$ of the preferred overground speed. The treadmill speed was gradually increased by $0.1 \mathrm{~km} \mathrm{~h}^{-1}$ until the volunteer reported that the speed was too fast. The speed was then gradually reduced at the same rate until the volunteer 
Table 1 Characteristics of the sample ( $n=35$ women)

\begin{tabular}{llll}
\hline & Fallers $(n=16)$ & Non-fallers $(n=19)$ & $p$ \\
\hline Age (years) & $69.6(8.1)$ & $66.1(6.2)$ & 0.1 \\
Weight (kg) & $66.8(9.2)$ & $65.3(13.6)$ & 0.6 \\
Height (m) & $1.51(0.06)$ & $1.54(0.05)$ & 0.2 \\
Preferred gait speed $\left(\mathrm{m} / \mathrm{s}^{-1}\right)$ & $0.9(0.1)$ & $0.9(0.1)$ & 0.6 \\
\hline
\end{tabular}

reported that the treadmill speed was too slow. This procedure was repeated three times. The mean of the speeds considered to be too fast and too slow was defined as the preferred treadmill gait speed. The volunteers walked at their preferred treadmill speed for $10 \mathrm{~min}$ to familiarize themselves with walking on the treadmill at that speed [18].

After familiarization, the subjects walked for three consecutive minutes on the treadmill, and the temporal gait parameters of stance time, swing time and stride time were calculated for this period. The subjects wore a safety harness connected to a safety rope attached to the ceiling to prevent falls during gait assessment. Footswitch sensors were placed under the feet of the volunteers on the heel, toe, base of the hallux and fifth metatarsal to determine the phases of gait.

\section{Data analysis}

Forty consecutive gait cycles were obtained during the treadmill walk and used for data analysis. Determining the start and end of the stance phase, swing phase and stride phase used data from the footswitch sensors. When a heel strike occurred, the footswitch signal changed from 0 to $5 \mathrm{mV}$. When toe off occurred, the footswitch signal returned from $5 \mathrm{mV}$ to baseline. From the onset and offset of the footswitch, it was possible to determine stride time, stance time and swing time. Figure 1 shows how the signals from the footswitch sensor were used to determine heel strike, toe off, stance phase and swing phase.

Six statistical methods were used to calculate heart rate variability and determine the variability in stance time, swing time and stride time intervals [19]: (1) SDNN represents the standard deviation of all the time intervals expressed in ms; (2) SDANN is the standard deviation of the means of these intervals taken every five strides and expressed in ms; (3) SDNNi represents the mean of the standard deviations of the intervals determined every five strides and expressed in ms; (4) the rMSSD is the rootmean-square of the differences between intervals expressed in $\mathrm{ms}$; (5) $\mathrm{CV}$ is the coefficient of variation calculated as the standard deviation of the intervals divided by the mean of the intervals expressed as a percentage; and (6) the triangular index is a geometric method calculated based on the construction of a histogram of the intervals, which has on the horizontal axis (the length of intervals) - the vertical axis is the frequency at which each interval occurred. The junction of the points of the histogram columns forms a triangle that expresses the variability of the intervals. The triangular index corresponds to the width of the base of the triangle.

The Pearson correlation statistic was used to correlate the different methods of variability analysis. Multivariate analysis of variance was performed to compare the variability scores of each method between fallers and nonfallers. Then, stepwise discriminant analysis was used to determine a predictor model of falls for stance time, swing time and stride time. The discriminant analysis also provided the specificity and sensitivity of each method in the identification of fallers. The highest values of sensitivity and specificity were used to determine the most significant predictor model. A cutoff score was determined to identify the limit value that can discriminate older fallers and nonfallers. The significance level was defined as $p<0.05$ for all tests.

\begin{tabular}{|c|c|c|c|c|c|}
\hline \multicolumn{3}{|c|}{ Signals } & Toe Off & \multicolumn{2}{|l|}{ Swing Phase } \\
\hline \multirow{5}{*}{$\frac{\text { th }}{\text { All }}$} & \multirow{3}{*}{$\begin{array}{rr}9 & -+\mathbf{i} \\
-0.0 \\
\end{array}$} & 50 & 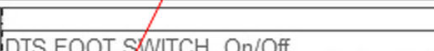 & \multirow[b]{2}{*}{$\square \longdiv { \square }$} & \multirow[b]{2}{*}{ 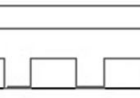 } \\
\hline & & $00-$ & $\square \square \square$ & & \\
\hline & & -5.0 & Heel Strike & ase & \\
\hline & Markers & & & & \\
\hline & & & $5.0000 \mathrm{~ms}$ & $10.0000 \mathrm{~ms}$ & $15.0000 \mathrm{~ms}$ \\
\hline
\end{tabular}

Fig. 1 Signal from sensors footswitch for the determination of start and end of the stance phase, swing phase and stride phase 


\section{Results}

Table 2 shows the Pearson's correlation coefficients between methods of analysis of variability for stance time, swing time and stride time. The SDNNi values were strongly and positively correlated with rMSSD in stance time, swing time and stride time. Despite smaller correlation coefficients, each of the other comparisons between variability measures elicited significant, positive correlations.

Significant differences existed between older fallers and non-fallers for some methods of variability analysis for stance time, swing time and stride time (Figs. 2, 3, 4). For stance time, there were significant differences between groups for SDNN, SDNNi, rMSSD and CV ( $p=0.000$, $p=0.043, p=0.030$ and $p=0.030$, respectively). For swing time, there was a significant difference between groups only for SDANN ( $p=0.047$ ). For step time, there were significant differences between groups for SDNN, SDNNi, rMSSD and triangle index $(p=0.026, p=0.039$, $p=0.041$ and $p=0.004$, respectively).

The magnitude of the significant differences between groups expressed in percentage for stance time for SDNN, SDNNi, rMSSD and CV was 295.0, 24.1, 25.6 and $29.6 \%$, respectively. For swing time, the magnitude of differences between groups for SDANN was $30.2 \%$. For stride time, the magnitude of significant differences between groups for

Table 2 Pearson's correlation coefficients between methods of analysis of variability in stance time, swing time and stride time

\begin{tabular}{|c|c|c|c|c|c|}
\hline & SDANN & SDNNi & rMSSD & $\mathrm{CV}$ & $\begin{array}{l}\text { Triangle } \\
\text { index }\end{array}$ \\
\hline \multicolumn{6}{|l|}{ Stance time } \\
\hline SDNN & 0.247 & 0.375 & 0.374 & 0.398 & 0.374 \\
\hline SDANN & 1 & $0.503 * *$ & $\mathrm{0.433}^{* *}$ & $0.357 *$ & 0.225 \\
\hline SDNNi & & 1 & $\mathbf{0 . 9 4 8} * *$ & $0.785 * *$ & $0.646 * *$ \\
\hline rMSSD & & & 1 & $0.814 * *$ & $0.635 * *$ \\
\hline $\mathrm{CV}$ & & & & 1 & $0.536 * *$ \\
\hline \multicolumn{6}{|l|}{ Swing time } \\
\hline SDNN & $0.720 * *$ & 0.714** & 0.708 & 0.710 & 0.348 \\
\hline SDANN & 1 & $\mathbf{0 . 5 0 7} * *$ & $0.528 * *$ & $0.612 * *$ & $0.495 * *$ \\
\hline SDNNi & & 1 & $0.947 * *$ & $0.768 * *$ & $0.593 * *$ \\
\hline rMSSD & & & 1 & $0.787 * *$ & $0.665 * *$ \\
\hline $\mathrm{CV}$ & & & & 1 & $0.384 *$ \\
\hline \multicolumn{6}{|l|}{ Stride time } \\
\hline SDNN & $0.665 * *$ & $0.923 * *$ & 0.917 & 0.803 & 0.742 \\
\hline SDANN & 1 & $0.400 *$ & $0.340 *$ & $0.764 * *$ & 0.710 \\
\hline SDNNi & & 1 & 0.948** & $0.660 * *$ & $0.591 * *$ \\
\hline rMSSD & & & 1 & $0.616 * *$ & $0.572 * *$ \\
\hline $\mathrm{CV}$ & & & & 1 & $0.623 * *$ \\
\hline
\end{tabular}

Significant correlations are in bold. $* p<0.05 ; * * p<0.01$
SDNN, SDNNi, rMSSD and triangle index was 21.2, 19.8, 19.1 and $27.0 \%$, respectively (Figs. 2, 3, 4).

The predictor models developed from the discriminant analysis for history of falls are shown in Table 3 for stance time, swing time and stride time. The analysis demonstrated that SDNN of stance time was the most significant predictor model for the correct identification of fallers and non-fallers $\quad(p=0.000, \quad$ sensitivity/specificity $=100 /$ $100 \%)$. The cutoff point for SDNN of stance time that discriminated older adults fallers and non-fallers was $0.102 \mathrm{~s}$. For swing time variability, the SDANN analysis was the strongest predictor $(p=0.047)$ of fall status, but had lower sensitivity (56\%) and specificity (78\%) than SDNN of stance time. Similarly, the triangle index was the strongest predictor ( $p=0.004$ ) of fall status using stride time variability with a lower sensitivity (50\%) and specificity $(91 \%)$ than SDNN of stance time.

\section{Discussion}

The purpose of this study was to identify the best temporal kinematic variability method to discriminate older fallers and non-fallers. We chose to use temporal gait parameters because previous studies demonstrated that the variability effect is greater for temporal gait characteristics than for spatial characteristics [12]. Thus, we tested the sensitivity and specificity to detect the fall status of three kinematic temporal parameters (stride time, stance time and swing time) most frequently used to study gait variability. The major novel finding is that the SDNN (standard deviation) of stance time is the best discriminant variable of older fallers and non-fallers. We also found a cutoff point of $0.102 \mathrm{~s}$ for SDNN of stance time, which may be useful to identify older adults at risk of falling using a simple and inexpensive footswitch system.

Sensitivity and specificity are statistical measures that allow researchers to test the ability of a predictor variable to screen a population for a specific health characteristic. For example, routine screening of blood pressure is used to detect atherosclerosis. Sensitivity describes the ability to correctly detect people with the disease, and specificity describes the ability to correctly identify people without the disease. In our cohort, the calculation of the SDNN of stance time had $100 \%$ sensitivity and specificity to discriminate older fallers and non-fallers.

We performed a simple walking test using an inexpensive footswitch device to assess gait variability in older adults. Our walking test was performed with older subjects walking on the treadmill after a previous habituation section. The entire test (subjects preparation, overground speed test, selection of preferred treadmill walking speed, 
Fig. 2 Average group

differences in the methods of analysis of variability in stance time. ${ }^{*} p<0.05$
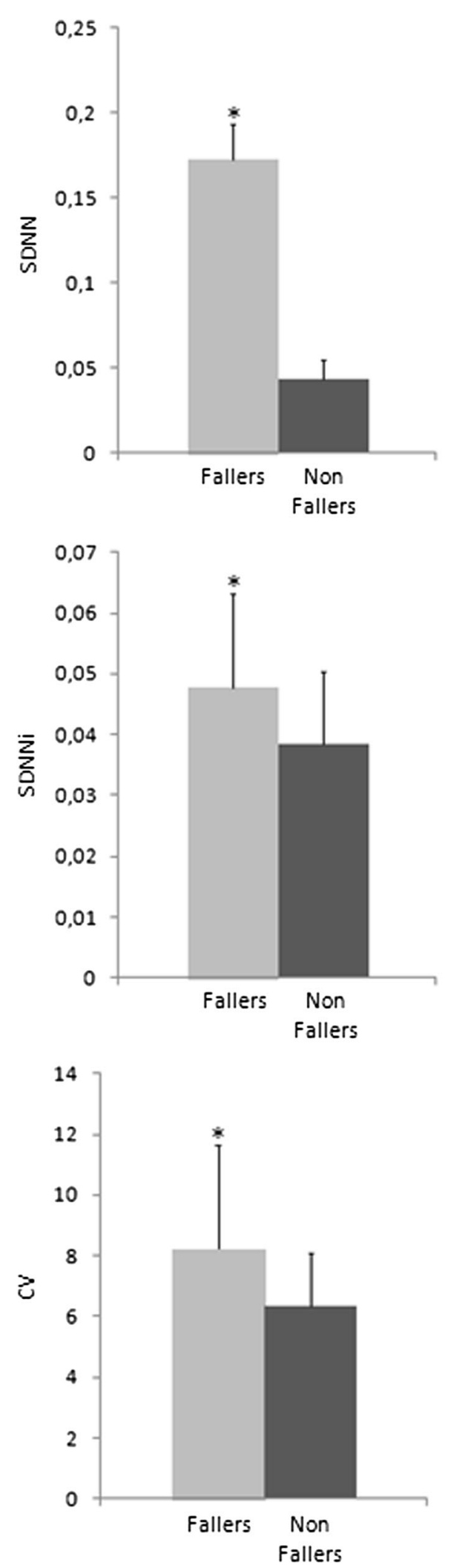
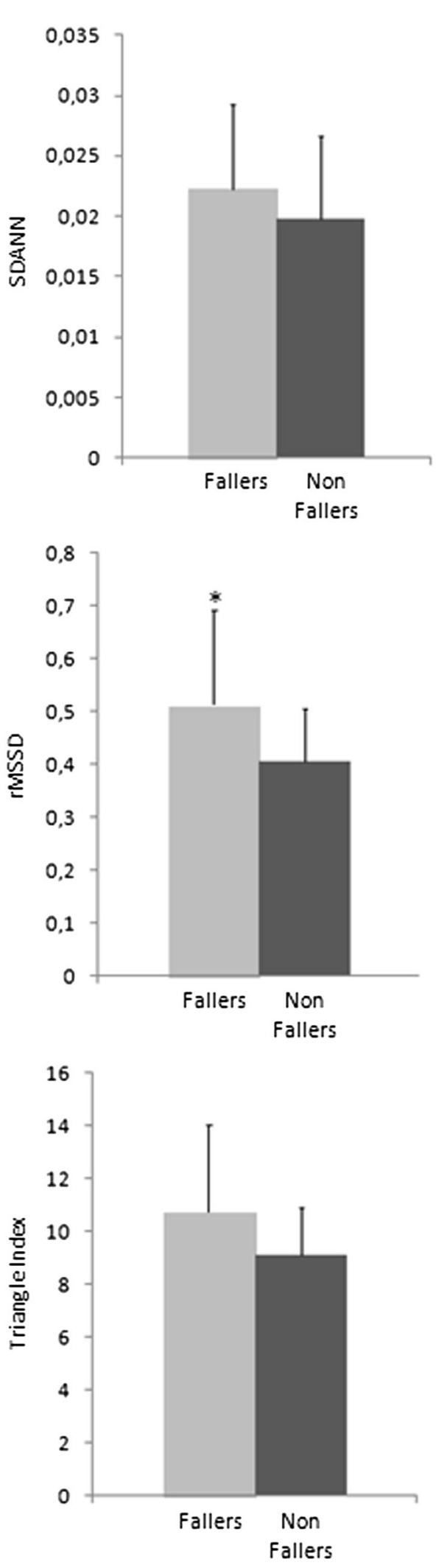

habituation and data collection) took about $30 \mathrm{~min}$. Assessing the variability in overground gait at the preferred walking speed could reduce the analysis time.
Measurement of the SDNN of stance time can therefore be useful for physical therapists, physicians and nursing home attendants to assess the risk of falls in older adults. In 
Fig. 3 Average group

differences in the methods of analysis of variability in swing time. ${ }^{*} p<0.05$
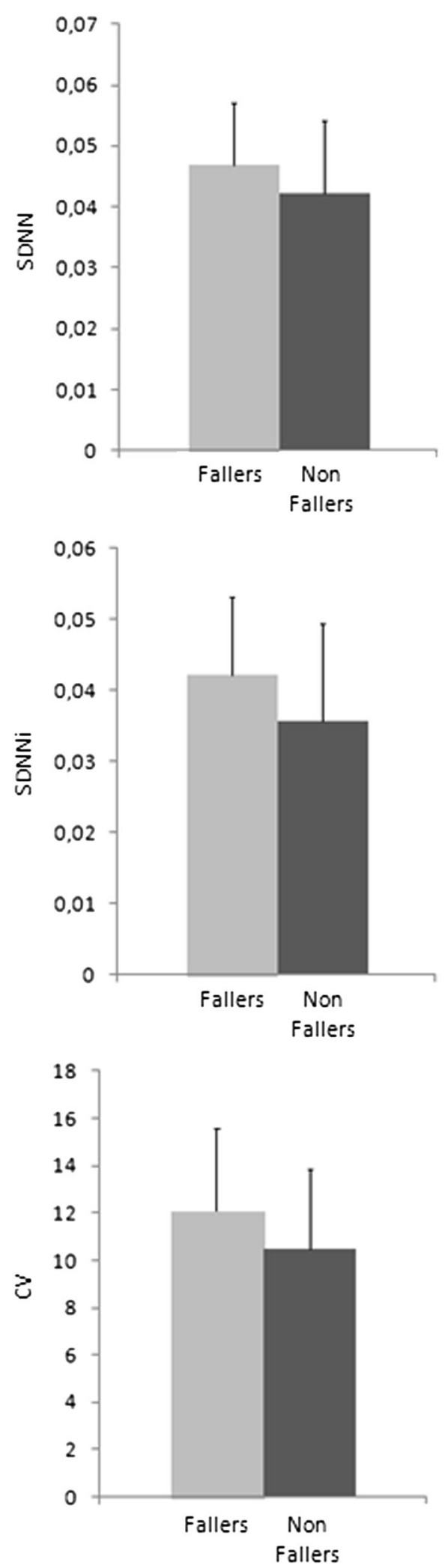
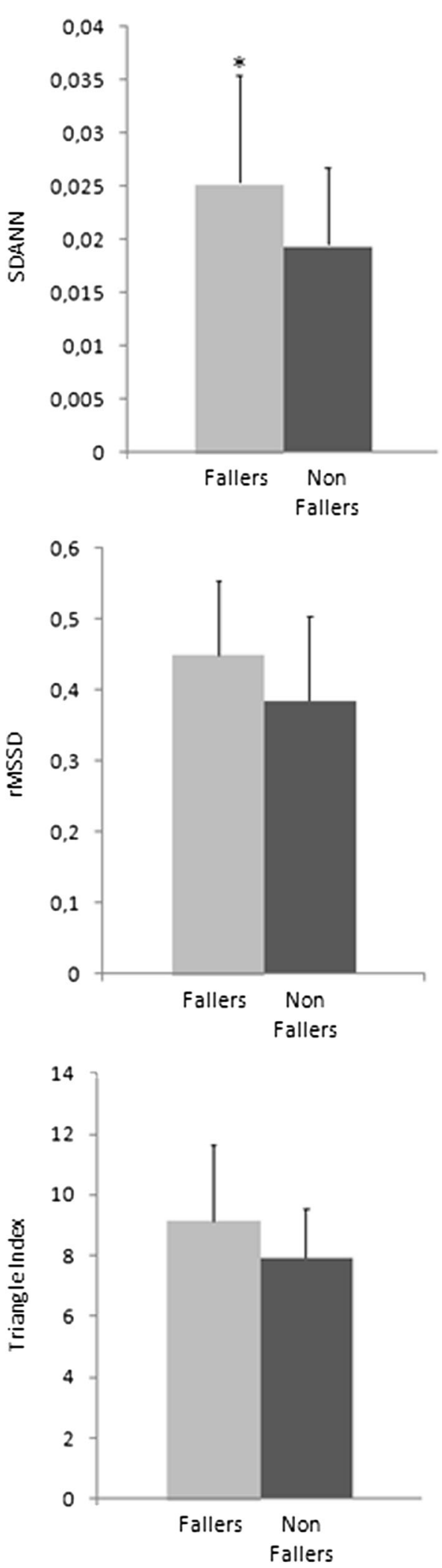
Fig. 4 Average group

differences in the methods of analysis of variability in stride time. ${ }^{*} p<0.05$
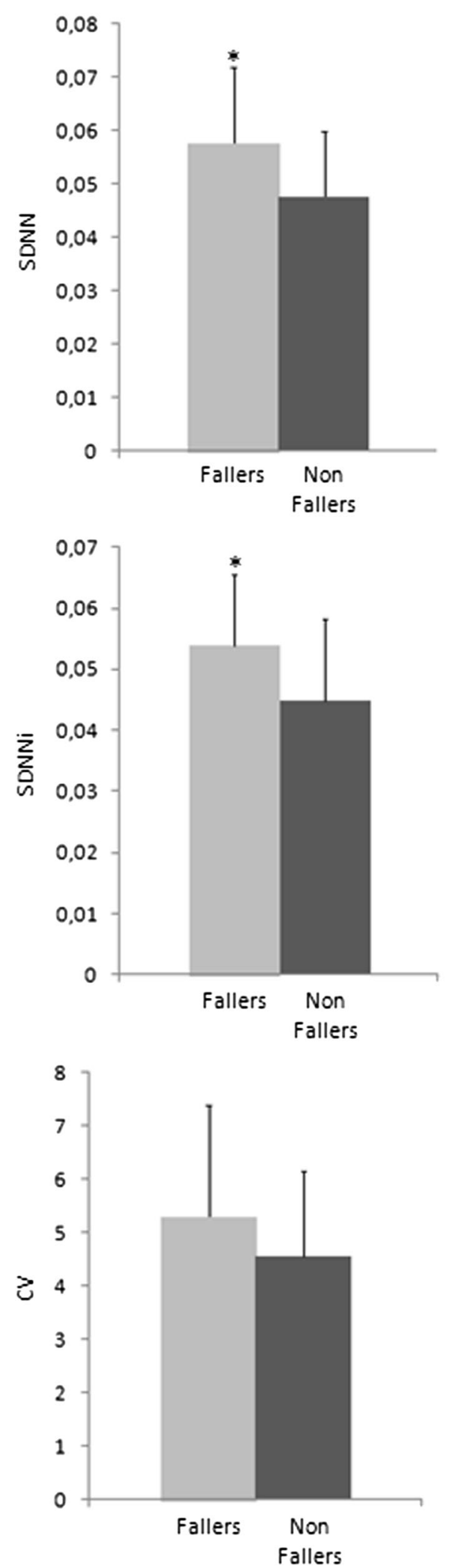
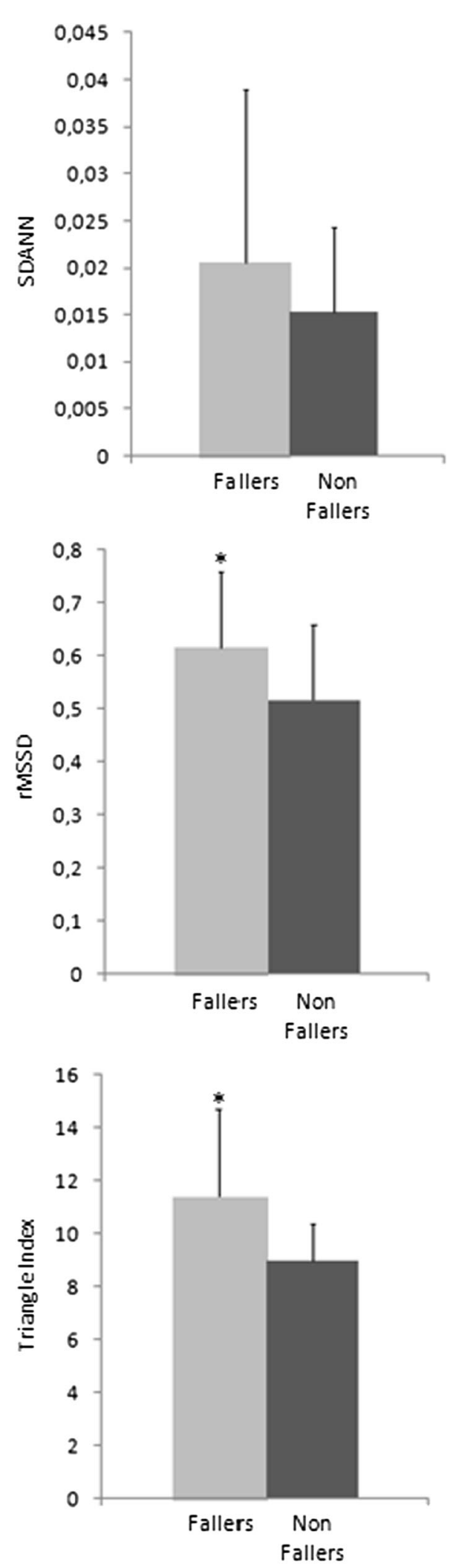
Table 3 Predictive variables result from stepwise discriminant analyses on phases of gait

\begin{tabular}{llll}
\hline & Predictive variable & $p$ & \multicolumn{1}{c}{$\begin{array}{c}\text { Discriminant analysis } \\
\text { (sensitivity/specificity \%) }\end{array}$} \\
\hline Stance time & SDNN & 0.000 & $100 / 100$ \\
Swing time & SDANN & 0.047 & $56 / 78$ \\
Stride time & Triangle index & 0.004 & $50 / 91$ \\
\hline
\end{tabular}

comparison with other devices that can be used to evaluate gait like accelerometers, gyroscopes, optical motion analysis, gait mats, and force plates [13], footswitch sensors are cheaper, easier to use and do not require excessive technical skill.

A previous study used physical assessment tests involving strength tests, to evaluate the best parameter capable of discriminating older fallers and non-fallers [20]. According to Hausdorff et al. [20], the maximum horizontal leg press strength discriminated fallers with $86 \%$ sensitivity and $100 \%$ specificity. Our method discriminates older fallers and non-fallers using the standard deviation of stance time (SDNN). It can better utility in discriminating older fallers and non-fallers than leg strength as demonstrated by Hausdorff et al. [20].

While strength measurements are widely used to assess mobility status and risk of falling in older adults, strength is one of the only several variables (e.g., vision, vestibular function, motor control) that influence gait performance. Therefore, the assessment of gait variability may possibly have a greater predictive ability to identify the risk of falls than strength.

Previous studies described that even when strength and gait speed are similar, the gait variability might differentiate older fallers and non-fallers [13, 21]. In accordance with these studies, our results showed that gait speed was similar between older fallers and non-fallers although the stride-to-stride changes were different between groups. Also similar to our results, Pijnappels et al. [21] demonstrated that older fallers had increased gait variability-not only in the period of one stride cycle, but also in the subphases within each cycle. With respect to this, a relatively increased stride-to-stride variability may reflect an unsteady gait, which increases the risk of falling [13, 21].

We tested six different methods to calculate gait variability. These are widely used to calculate heart rate variability. The SDNN is calculated from the standard deviation of all time intervals [19], and the percent of variability was determined to find the cutoff point to discriminate older fallers and non-fallers. Of the tested methods, SDNN was the only one that considers the standard deviation alone for calculation of gait variability. We suspect that this approach had higher sensitivity and specificity than the other methods that combine standard deviation and average values (SDANN, SDNNi, rMSSD and $\mathrm{CV}$ ) because the use of the average may smooth the data and reduces the data dispersion.

Despite these gait variability measures, our interrelated use of other methods to calculate gait variability is not necessary because SDNN alone was a strong predictor of fall status. Also, the use of SDNN to calculate gait variability in older adults can be an important clinical tool because it is easy to calculate and does not require any sophisticated software for data analysis.

Discriminant analysis provided a cutoff point for SDNN (0.102 s) during stance time that can be used to discriminate older adults fallers and non-fallers much like a systolic blood pressure above $140 \mathrm{mmHg}$ suggests hypertension and the possibility of cardiovascular disease. The cutoff point value may therefore be a useful benchmark for the clinical assessment of fall risk-particularly for older adults without a history of falls or those who cannot remember fall episodes.

Older adults with limited mobility may not yet have fallen because they are not exposed to hazardous situations during daily activity as a result of their low levels of activity. It may therefore be possible to use the SDNN of stance time to identify those at risk of falling and intervene with modifications to the environment (remove rugs, add railings in the home) as well as adding balance, strength and motor control exercise strategies to mitigate fall risk [22].

Our findings also demonstrated that the variability of stance time was a better discriminative variable than the variability of swing time or stride time. Beyond the SDNN of stance time, the SDANN of swing time and triangle index of stride time are discriminant parameters in older fallers and non-fallers. However, the sensitivity of both methods (around of $50 \%$ ) was low and is only correct in half of the cases-this is not acceptable as a tool in programs to prevent falls. Thus, the ability of the stance time variability to discriminate older fallers and non-faller may be related to the biomechanical demands involved in this gait phase.

During the initial phase of stance, muscles are activated to maintain joint stability, while the ground reaction forces are absorbed [23]. During the terminal phase of stance, the plantar flexors are recruited to accelerate the center of mass to perform the next step, while the hip and trunk muscles are activated to maintain balance [23]. Thus, the stance is 
the gait phase that requires the most strength, balance and motor control during walking gait. In this regard, older adult fallers may have higher gait variability for stance time because this population has lower strength and poorer balance than non-fallers [24].

The limitation is that the gait variability cutoff point found here may be specific for this specific sample of women who are healthy, physically fit and normally mobile. Thus, extrapolation of our findings should be made with caution. Also, we tested the ability of the variability to discriminate older fallers and non-fallers walking on the treadmill. Thus, the results may have relation to the nature of the treadmill walking task. Future studies must be conducted to identify the ability of using the SDNN of stance time to predict fall events. Also, investigations with the fastest and slowest gait speed may elucidate the ability of SDNN to discriminate older fallers and non-fallers even during speed-induced gait perturbation.

\section{Conclusion}

This study tested six mathematical methods to determine gait variability from three different kinematic gait parameters and concluded that the standard deviation of stance time is the kinematic gait that has the best ability to discriminate older fallers from non-fallers. This easily obtained measure of gait variability may therefore be an important clinical tool to assess risk of falls in older adults. However, the utility of this method to predict falls should be evaluated prospectively in a larger and more diverse sample.

Acknowledgments Funding for this study was provided by Sao Paulo Research Foundation (FAPESP Process Number: 2011/11639-7.

\section{Compliance with ethical standards}

Conflict of interest statement The authors declare do not have any conflict of interest.

Statement of human and animal rights The present study was conducted inaccordance of human rights and approved bylocal ethics comittee.

Informedconsent All volunteers signed the informed consent form.

\section{References}

1. Schulz BW, Lloyd JD, William E (2010) The effects of everyday concurrent task on overground minimum toe clearance and gait parameters. Gait Post 32:18-22

2. Millat AJ, Watson WL, Monger C et al (2011) Prevalence, circumstances and consequences of falls among community- dwelling older people: results of the 2009 NSW Falls Prevention Baseline Survey. NSW Pub Health Bull 22:43-48

3. Van Dieen JH, Pijnappels M (2008) Falls in older people. J Electromyogr Kinesiol 18:169-171

4. Bassey EJ (1997) Physical capabilities, exercise and aging. Rev Clin Gerontol 20:289-297

5. Tinetti M, Speechley M (1989) Prevention of falls among the elderly. N Engl J Med 320:1055-1059

6. Kirkwood RN, Trede RG, Moreira BS et al (2011) Decreased gastrocnemius temporal muscle activation during gait in elderly women with history of recurrent falls. Gait Post 34:60-64

7. Callisaya ML, Blizzard L, Schmidt MD et al (2011) Ageing and gait variability - a population-based clinimetric properties. Gait Post 34:443-450

8. Owings TM, Grabiner MD (2004) Variability of step kinematics in young and older adults. Gait Post 20:26-29

9. Chen CJ, Chou LS (2010) Center of mass position relative to the ankle during walking: a clinically feasible detection method for gait imbalance. Gait Post 31:391-393

10. Donoghue OA, Cronin H, Savva GM et al (2013) Effects of fear of falling and activity restriction on normal and dual task walking in community dwelling older adults. Gait Post 38:120-124

11. Hausdorff JM, Rios DA, Edelberg HK (2011) Gait variability and fall risk in community-living older adults: a 1-year prospective study. Arch Phys Med Rehabil 82:1050-1055

12. Lord S, Galna B, Verghese J et al (2013) Independent domains of gait in older adults and associated motor and nonmotor attributes: validation of a factor analysis approach. J Gerontol A Biol Sci Med Sci 67:820-827

13. Hausdorff JM (2005) Gait variability: methods, modeling and meaning. J Neuroeng Rehabil 2:1-9

14. Brach JS, Studenski S, Perera S et al (2007) Gait variability and the risk of incident mobility disability in community-dwelling older adults. J Gerontol A Biol Sci Med Sci 9:983-988

15. Siqueira FV, Facchini LA, Silveira DS et al (2011) Prevalence of falls in elderly in Brazil: a countrywide analysis. Cad Saude Publ 27:1819-1826

16. Tucker MG, Kavanagh JJ, Morison S et al (2010) What are the relations between voluntary postural sway measures and fallshistory status in community-dwelling older adults? Arch Phys Med Rehabil 91:750-758

17. Watt JR, Franz JR, Jackson K et al (2010) A three-dimensional kinematic and kinetic comparison of overground and treadmill walking in healthy elderly subjects. Clin Biomech 25:444-449

18. Dingwell JB, Marin LC (2006) Kinematic variability and local dynamic stability of upper body motions when walking at different speeds. J Biomech 39:444-452

19. Pumprla J, Howorka K, Groves D et al (2002) Functional assessment of heart rate variability: physiological basis and practical applications. Int J Cardiol 84:1-14

20. Hausdorff JM, Edelberg HK, Mitchell SL et al (1997) Increased gait unsteadiness in community-dwelling elderly fallers. Arch Phys Med Rehabil 78:278-283

21. Pijnappels M, Van Der Burg JCE, Reeves ND et al (2008) Identification of elderly fallers by muscle strength measures. Eur J Appl Physiol 102:585-592

22. Zenewton ASG, Conesa AG (2008) Factores de riesgo de caídas em ancianos: revisión sistemática. Rev Saúde Pub 42:946-956

23. LaRoche DP, Millet ED, Kralian RJ (2011) Low strength is related to diminished ground reaction forces and walking performance in older women. Gait Post 33:668-672

24. Hausdorff JM (2007) Gait dynamics, fractals and falls: finding meaning in the stride-to-stride fluctuations of human walking. Human Mov Sci 26:555-589 